\title{
Design of the NASA Robonaut Hand
}

\author{
C. S. Lovchik, H. A. Aldridge \\ Robotics Technology Branch \\ NASA Johnson Space Center \\ Houston, Texas 77058 \\ lovchik@jsc.nasa.gov, haldridg@ems.jsc.nasa.gov \\ Fax: 281-244-5534
}

\author{
M. A. Diftler \\ Automation and Robotics Department \\ Lockheed Martin \\ Houston, Texas 77058 \\ diftler@jsc.nasa.gov \\ Fax: 281-244-5534
}

\begin{abstract}
The design of a highly anthropomorphic human scale robot hand for space based operations is described. This five finger hand combined with its integrated wrist and forearm has fourteen independent degrees of freedom. The device approximates very well the kinematics and required strength of an astronaut's hand when operating through a pressurized space suit glove. The mechanisms used to meet these requirements are explained in detail along with the design philosophy behind them. Integration experiences reveal the challenges associated with obtaining the required capabilities within the desired size. The initial finger control strategy is presented along with examples of obtainable grasps.
\end{abstract}

\section{Introduction}

The requirements for extra-vehicular activity (EVA) onboard the International Space Station (ISS) are expected to be considerable. These maintenance and construction activities are expensive and hazardous. Astronauts must prepare extensively before they may leave the relative safety of the space station, including pre-breathing at space suit air pressure for up to 4 hours. Once outside, the crew person must be extremely cautious to prevent damage to the suit.

The Robotic Systems Technology Branch at the NASA Johnson Space Center is currently developing robot systems to reduce the EVA burden on space station crew and also to serve in a rapid response capacity. One such system, Robonaut is being designed and built to interface with external space station systems that only have human interfaces. To this end, the Robonaut hand [1] provides a high degree of anthropomorphic dexterity ensuring a compatibility with many of these interfaces.

Many ground breaking dexterous robot hands [2-7] have been developed over the past two decades. These devices make it possible for a robot manipulator to grasp and manipulate objects that are not designed to be robotically compatible. While several grippers [8-12] have been designed for space use and some even tested in space $[8,9,11]$, no dexterous robotic hand has been flown in EVA conditions. The Robonaut Hand is one of several hands $[13,14]$ under development for space EVA use and is closest in size and capability to a suited astronaut's hand.

\section{Design and Control Philosophy}

The requirements for interacting with planned space station EVA crew interfaces and tools provided the starting point for the Robonaut Hand design [1]. Both power (enveloping) and dexterous grasps (finger tip) are required for manipulating EVA crew tools. Certain tools require single or multiple finger actuation while being firmly grasped. A maximum force of $20 \mathrm{lbs}$ and torque of 30 in-lbs are required to remove and install EVA orbital replaceable units (ORUs) [15]. All EVA tools and ORUs must be retained in the event of a power loss.

It is possible to either build interfaces that will be both robotically and EVA compatible or build a series of robot tools to interact with EVA crew interfaces and tools. However, both approaches are extremely costly and will of course add to a set of space station tools and interfaces that are already planned to be quite extensive. The Robonaut design will make all EVA crew interfaces and tools robotically compatible by making the robot's hand EVA compatible.

EVA compatibility is designed into the hand by reproducing, as closely as possible, the size, kinematics, and strength of the space suited astronaut hand and wrist. The number of fingers and the joint travel reproduce the workspace for a pressurized suit glove. The Robonaut Hand reproduces many of the necessary grasps needed for interacting with EVA interfaces. Staying within this size envelope guarantees that the Robonaut Hand will be able to fit into all the required places. Joint travel for the wrist pitch and yaw is designed to meet or exceed the human hand in a pressurized glove. The hand and wrist parts are 
sized to reproduce the necessary strength to meet maximum EVA crew requirements.

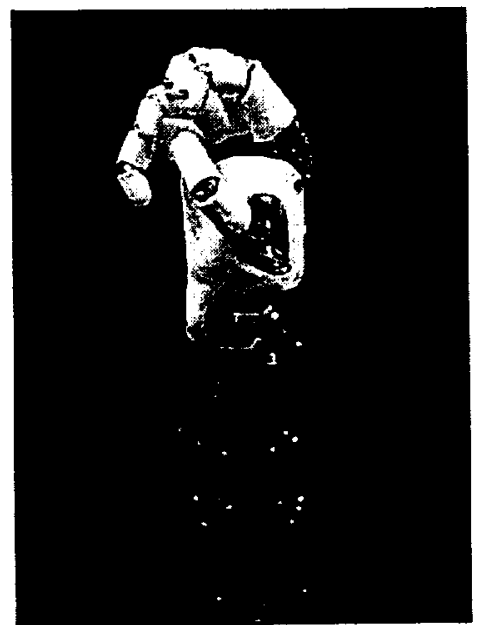

Figure 1: Robonaut Hand

Control system design for a dexterous robot hand manipulating a variety of tools has unique problems. The majority of the literature available, summarized in $[2,16]$, pertains to dexterous manipulation. This literature concentrates on using three dexterous fingers to obtain force closure and manipulate an object using only fingertip contact. While useful, this type of manipulation does not lend itself to tool use. Most EVA tools are best used in an enveloping grasp. Two enveloping grasp types, tool and power, must be supported by the tool-using hand in addition to the dexterous grasp. Although literature is available on enveloping grasps [17], it is not as advanced as the dexterous literature. The main complication involves determining and controlling the forces at the many contact areas involved in an enveloping grasp.

While work continues on automating enveloping grasps, a teleoperation control strategy has been adopted for the Robonaut hand. This method of operation was proven with the NASA DART/FITT system [18]. The DART/FITT system utilizes Cyberglove ${ }^{\circledR}$ virtual reality gloves, worn by the operator, to control Stanford/JPL hands to successfully perform space relevant tasks.

\subsection{Space Compatibility}

EVA space compatibility separates the Robonaut Hand from many others. All component materials meet outgassing restrictions to prevent contamination that could interfere with other space systems. Parts made of different materials are toleranced to perform acceptably under the extreme temperature variations experienced in EVA conditions. Brushless motors are used to ensure long life in a vacuum. All parts are designed to use proven space lubricants.

\section{Design}

The Robonaut Hand (figure 1) has a total of fourteen degrees of freedom. It consists of a forearm which houses the motors and drive electronics, a two degree of freedom wrist, and a five finger, twelve degree of freedom hand. The forearm, which measures four inches in diameter at its base and is approximately eight inches long, houses all fourteen motors, 12 separate circuit boards, and all of the wiring for the hand.

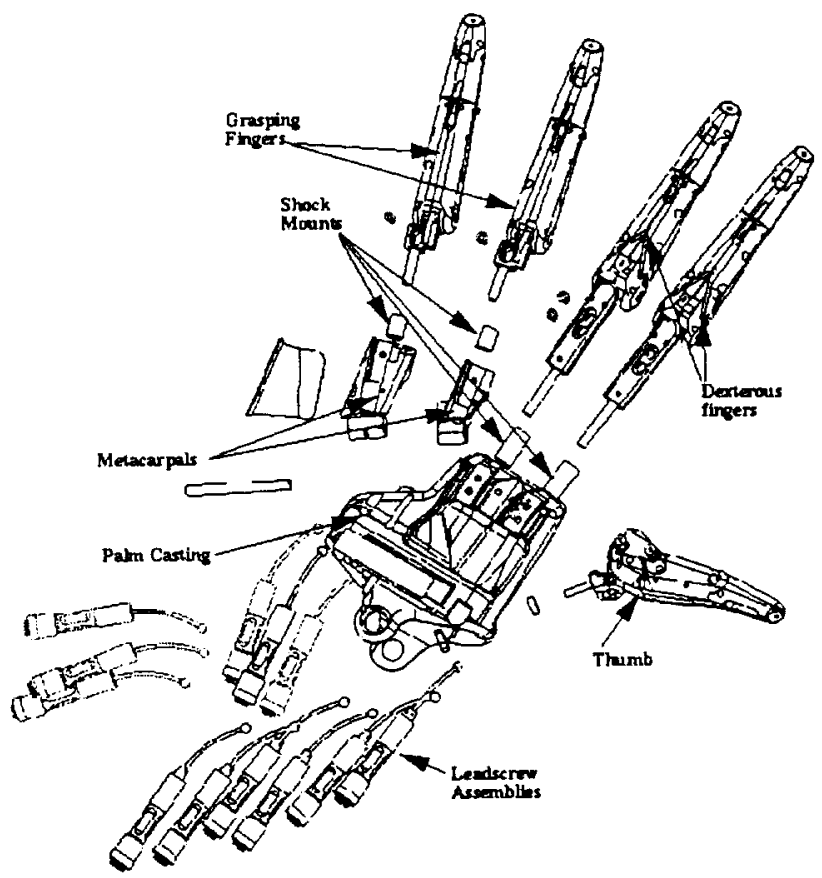

Figure 2: Hand components

The hand itself is broken down into two sections (figure 2): a dexterous work set which is used for manipulation, and a grasping set which allows the hand to maintain a stable grasp while manipulating or actuating a given object. This is an essential feature for tool use [13]. The dexterous set consists of two three degree of freedom fingers (pointer and index) and a three degree of freedom opposable thumb. The grasping set consists of two, one degree of freedom fingers (ring and pinkie) and a palm degree of freedom. All of the fingers are shock mounted into the palm (figure 2 ).

In order to match the size of an astronaut's gloved hand, the motors are mounted outside the hand, and mechanical power is transmitted through a flexible drive train. Past hand designs $[2,3]$ have used tendon drives which utilize complex pulley systems or sheathes, both of which pose serious wear and reliability problems when used in the EVA space environment. To avoid the problems associated with tendons, the hand uses flex shafts to 
transmit power from the motors in the forearm to the fingers. The rotary motion of the flex shafts is converted to linear motion in the hand using small modular leadscrew assemblies. The result is a compact yet rugged drive train.

Overall the hand is equipped with forty-three sensors not including tactile sensing. Each joint is equipped with embedded absolute position sensors and each motor is equipped with incremental encoders. Each of the leadscrew assemblies as well as the wrist ball joint links are instrumented as load cells to provide force feedback.

\subsection{Finger Drive Train}

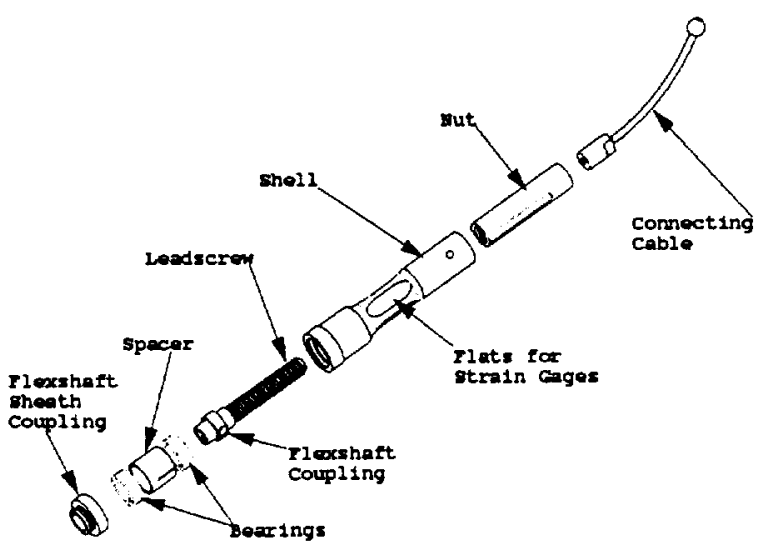

Figure 3: Finger leadscrew assembly

The finger drive consists of a brushless DC motor equipped with an encoder and a 14 to 1 planetary gear head. Coupled to the motors are stainless steel high flexibility flexshafts. The flexshafts are kept short in order to minimize vibration and protected by a sheath consisting of an open spring covered with Teflon. At the distal end of the flex shaft is a small modular leadscrew assembly (figure 3). This assembly converts the rotary motion of the flexshaft to linear motion. The assembly includes: a leadscrew which has a flex shaft connection and bearing seats cut into it, a shell which is designed to act as a load cell, support bearings, a nut with rails that mate with the shell (in order to eliminate off axis loads), and a short cable length which attaches to the nut. The strain gages are mounted on the flats of the shell indicated in figure 3 .

The top of the leadscrew assemblies are clamped into the palm of the hand to allow the shell to stretch or compress under load, thereby giving a direct reading of force acting on the fingers. Earlier models of the assembly contained an integral reflective encoder cut into the leadscrew. This configuration worked well but was eliminated from the hand in order to minimize the wiring in the hand.

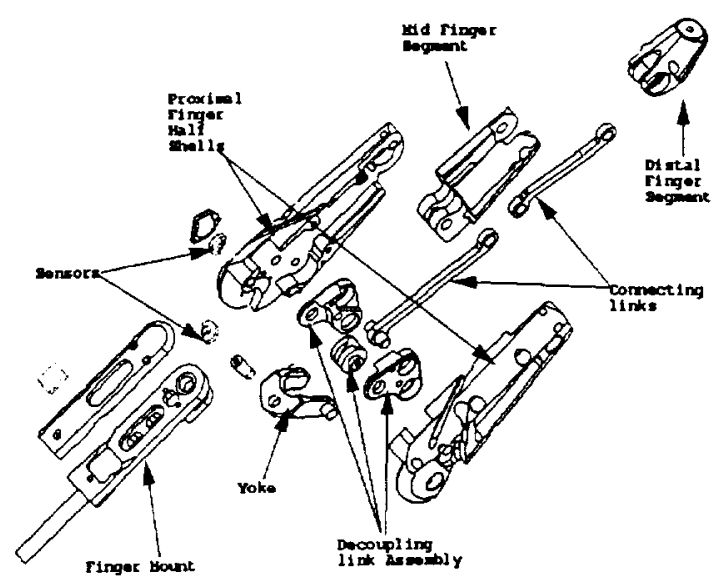

Figure 4: Dexterous finger

\subsection{Dexterous Fingers}

The three degree of freedom dexterous fingers (figure 4) include the finger mount, a yoke, two proximal finger segment half shells, a decoupling link assembly, a mid finger segment, a distal finger segment, two connecting links, and springs to eliminate backlash (not shown in figure).

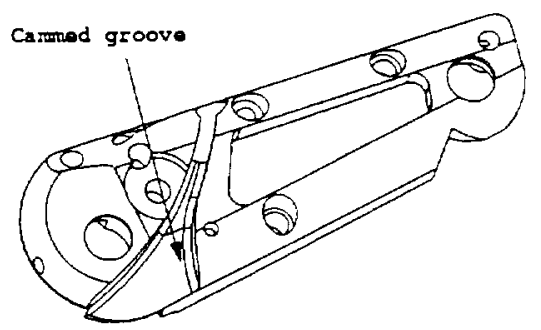

Figure 5 Finger base cam

The base joint of the finger has two degrees of freedom: yaw (+ $1-25$ degrees) and pitch (100 degrees). These motions are provided by two leadscrew assemblies that work in a differential manner. The short cables that extend from the leadscrew assemblies attach into the cammed grooves in the proximal finger segments half shells (figure 5). The use of cables eliminates a significant number of joints that would otherwise be needed to handle the two degree of freedom base joint. The cammed grooves control the bend radius of the connecting cables from the leadscrew assemblies (keeping it larger to avoid stressing the cables and allowing oversized cables to be used). The grooves also allow a nearly constant lever arm to be maintained throughout the full range of finger motion. Because the connecting cables are kept short (approximately 1 inch) and their bend radius is controlled (allowing the cables to be relatively large in diameter (.07 inches)), the cables act like stiff rods in the working direction (closing toward the 
palm) and like springs in the opposite direction. In other words, the ratio of the cable length to its diameter is such that the cables are stiff enough to push the finger open but if the finger contacts or impacts an object the cables will buckle, allowing the finger to collapse out of the way.

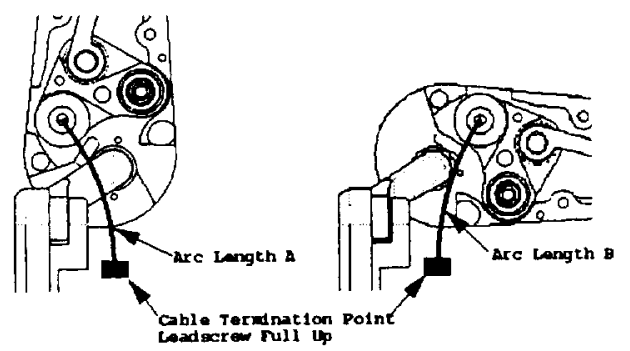

Figure 6: Decoupling link

The second and third joints of the dexterous fingers are directly linked so that they close with equal angles. These joints are driven by a separate leadscrew assembly through a decoupling linkage (figure 6). The short cable on the leadscrew assembly is attached to the pivoting cable termination in the decoupling link. The flex in the cable allows the actuation to pass across the two degree of freedom base joint, without the need for complex mechanisms. The linkage is designed so that the arc length of the cable is nearly constant regardless of the position of the base joint (compare arc $\mathrm{A}$ to arc $\mathrm{B}$ in figure 6). This makes the motion of distal joints approximately independent of the base joint.

\subsection{Grasping Fingers}

The grasping fingers have three pitch joints each with 90 degrees of travel. The fingers are actuated by one leadscrew assembly and use the same cam groove (figure 5 ) in the proximal finger segment half shell as with the manipulating fingers. The 7-bar finger linkage is similar to that of the dexterous fingers except that the decoupling link is removed and the linkage ties to the finger mount (figure 7). In this configuration each joint of the finger closes down with approximately equal angles. An alternative configuration of the finger that is currently being evaluated replaces the distal link with a stiff limited travel spring to allow the finger to better conform while grasping an object.

\subsection{Thumb}

The thumb is key to obtaining many of the grasps required for interfacing with EVA tools. The thumb shown in figure 2 has a proximal and distal segment and is similar in design to the dexterous fingers but has significantly more yaw travel and a hyper extended pitch. The thumb is also mounted to the palm at such an angle that the increase in range of motion results in a reasonable emulation of human thumb motion. This type of mounting enables the hand to perform grasps that are not possible with the common practice of mounting the thumb directly opposed to the fingers $[2,3,14]$. The thumb base joint has 70 degrees of yaw and 110 degrees of pitch. The distal joint has 80 degrees of pitch.

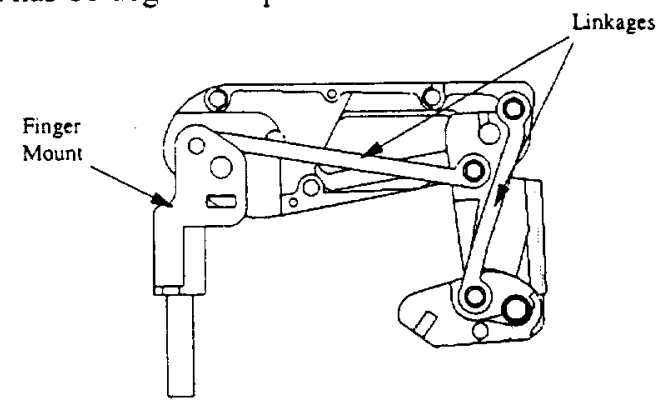

Figure 7: Grasping Finger

The actuation of the base joint is the same as the dexterous fingers with the exception that cammed detents have been added to keep the bend radius of the cable large at the extreme yaw angles. The distal segment of the thumb is driven through a decoupling linkage in a manner similar to that of the manipulating fingers. The extended yaw travel of the thumb base makes complete distal mechanical decoupling difficult. Instead the joints are decoupled in software.

\subsection{Palm}

The palm mechanism (figure 8 ) provides a mount for the two grasping fingers and a cupping motion that enhances stability for tool grasps. This allows the hand to grasp an object in a manner that aligns the tool's axis with the forearm roll axis. This is essential for the use of many common tools, like screwdrivers. The mechanism includes two pivoting metacarpals, a common shaft, and two torsion springs.

The grasping fingers and their leadscrew assemblies mount into the metacarpals. The metacarpals are attached to the palm on a common shaft. The first torsion spring is placed between the two metacarpals providing a pivoting force between the two. The second torsion spring is placed between the second metacarpal and the palm, forcing both of the metacarpals back against the palm. The actuating leadscrew assembly mounts into the palm and the short cable attaches to the cable termination on the first metacarpal. The torsion springs are sized such that as the leadscrew assembly pulls down the first metacarpal, 
the second metacarpal follows at roughly half the angle of the first. In this way the palm is able to cup in a way similar to that of the human hand without the fingers colliding.

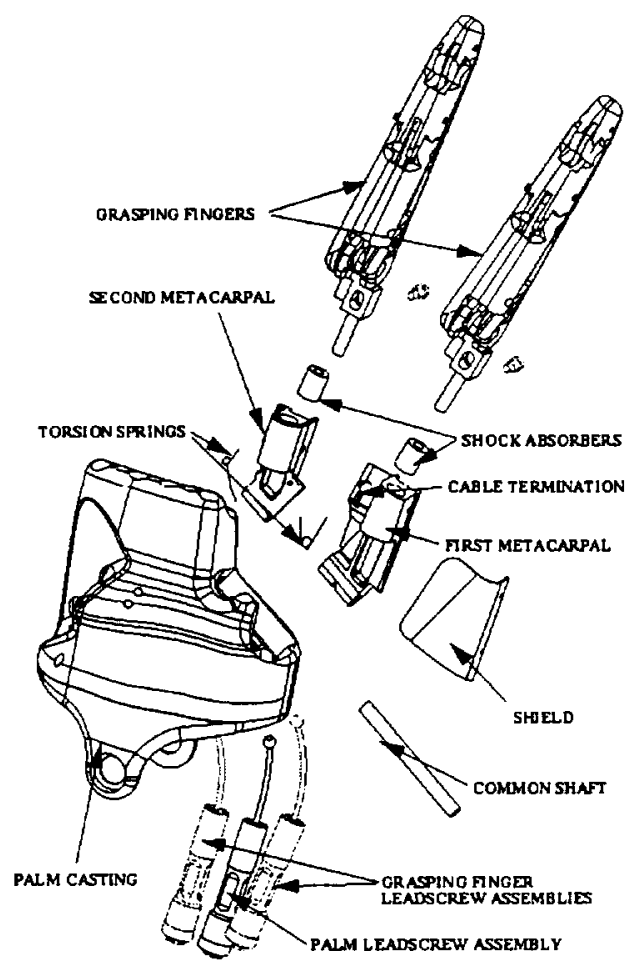

Figure 8: Palm mechanism

The fingers are mounted to the palm at slight angles to each other as opposed to the common practice of mounting them parallel to each other. This mounting allows the fingers to close together similar to a human hand. To further improve the reliability and ruggedness of the hand, all of the fingers are mounted on shock loaders. This allows them to take very high impacts without incurring damage.

\subsection{Wrist/Forearm Design}

The wrist (figure 9) provides an unconstrained pass through to maximize the bend radii for the finger flex shafts while approximating the wrist pitch and yaw travel of a pressurized astronaut glove. Total travel is $+/-70$ degrees of pitch and $+/-30$ degrees of yaw. The two axes intersect with each other and the centerline of the forearm roll axis. When connected with the Robonaut Arm [19], these three axes combine at the center of the wrist cuff yielding an efficient kinematic solution. The cuff is mounted to the forearm through shock loaders for added safety.
The wrist is actuated in a differential manner through two linear actuators (figure 9). The linear actuators consist of a slider riding in recirculating ball tracks and a custom, hollow shaft brushless DC motor with an integral ballscrew. The actuators attach to the palm through ball joint links, which are mounted in the pre-loaded ball sockets.

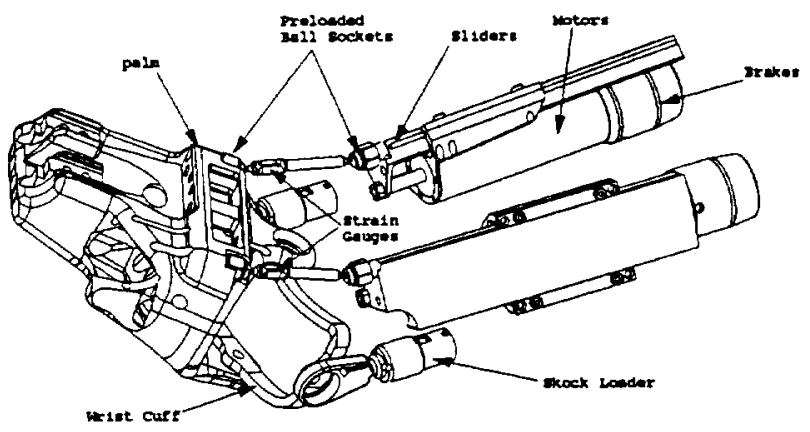

Figure 9 Wrist mechanism

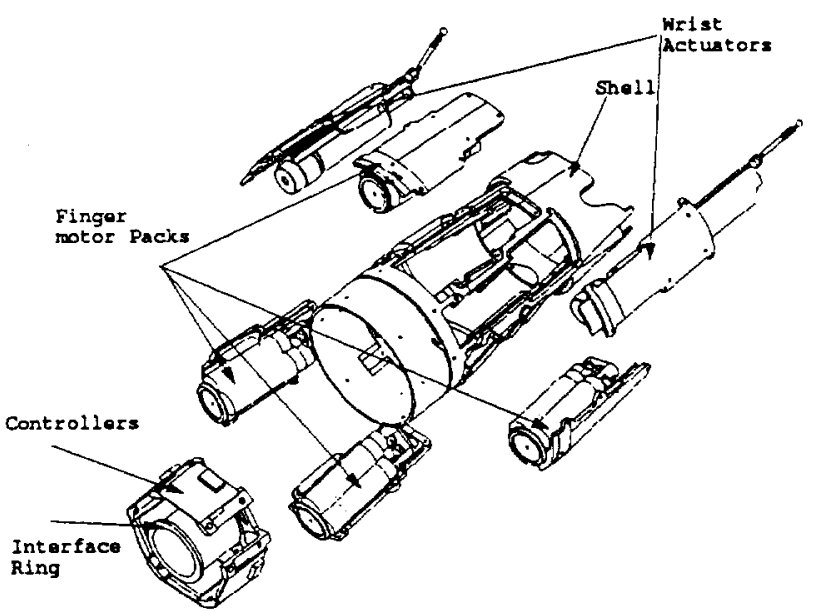

Figure 10: Forearm

The forearm is configured as a ribbed shell with six cover plates. Packaging all the required equipment in an EVA forearm size volume is a challenging task. The six cover plates are skewed at a variety of angles and keyed mounting tabs are used to minimize forearm surface area. Mounted on two of the cover plates are the wrist linear actuators, which fit into the forearm symmetrically to maintain efficient kinematics. The other four cover plate provides mounts for clusters of three finger motors (Figure 10). Symmetry is not required here since the flex shafts easily bend to accommodate odd angles. The cover plates are also designed to act as heat sinks. Along with the motors, custom hybrid motor driver chips are mounted to the cover plates. 


\section{Integration Challenges}

As might be expected, many integration challenges arose during hand prototyping, assembly and initial testing. Some of the issues and current resolutions follow.

Many of the parts in the hand use extremely complex geometry to minimize the part count and reduce the size of the hand. Fabrication of these parts was made possible by casting them in aluminum directly from stereo lithography models. This process yields relatively high accuracy parts at a minimal cost. The best example of this is the palm, which has a complex shape, and over 50 holes in it, few of which are orthogonal to each other.

Finger joint control is achieved through antagonistic cable pairs for the yaw joints and pre-load springs for the pitch joints. Initially, single compression springs connected through ball links to the front of the dexterous fingers applied insufficient moment to the base joints at the full open position. Double tension springs connected to the backs of the fingers improved pre-loading over more of the joint range. However, desired pre-loading in the fully open position resulted in high forces during closing. Work on establishing the optimal pre-load and making the preload forces linear over the full range is under way.

The finger cables have presented both mechanical mounting and mathematical challenges. The dexterous fingers use single mounting screws to hold the cables in place while avoiding cable pinch. This configuration allows the cables to flex during finger motion and yields a reasonably constant lever arm. However assembly with a single screw is difficult especially when evaluating different cable diameters. The thumb uses a more secure lock that includes a plate with a protrusion that securely presses down on the cable in its channel. The trade between these two techniques is continuing. Similar cable attachment devices are also evolving for the other finger joints.

The cable flexibility makes closed form kinematics difficult. The bend of the cable at the mounting points as the finger moves is not easy to model accurately. Any closed form model requires simplifying assumptions regarding cable bending and moving contact with the finger cams. A simpler solution that captures all the relevant data employs multi-dimensional data maps that are empirically obtained off-line. With a sufficiently high resolution these maps provide accurate forward and inverse kinematics data.

The wrist design (figure 9) evolved from a complex multibar mechanism to a simpler two-dimensional slider crank hook joint. Initially curved ball links connected the sliders to the palm with cams that rotated the links to avoid the wrist cuff during pitch motion. After wrist cuff and palm redesign, the present straight ball links were achieved.

The finger leadscrews are non-backdrivable and in an enveloping grasp ensure positive capture in the event of a power failure. If power can not be restored in a timely fashion, it may be necessary for the other Robonaut hand [19] or for an EVA crew person to manually open the hand. An early hand design incorporated a simple back out ring that through friction wheels engaged each finger drive train and slowly opened each finger joint. While this works well in the event of a power failure, experiments with the coreless brushless DC motors revealed a problem when a motor fails due to overheating. The motor winding insulation heats up, expands and seizes the motor, preventing back-driving. A new contingency technique for opening the hand that will accommodate both motor seizing and power loss is being investigated.

\section{Initial Finger Control Design and Test}

Before any operation can occur, basic position control of the Robonaut hand joints must be developed. Depending on the joint, finger joints are controlled either by a single motor or an antagonistic pair of motors. Each of these motors is attached to the finger drive train assembly shown in figure 3. A simple PD controller is used to perform motor position control tests. When the finger joint is unloaded, position control of the motor drive system is simple. When the finger is loaded, two mechanical effects influence the drive system dynamics. The flex shaft, which connects the motor to the lead screw, winds up and acts as a torsional spring. Although adding an extra system dynamic, the high ratio of the lead screw sufficiently masks the position error caused by the state of the flex shaft for teleoperated control.

The second effect during loading is the increased frictional force in the lead screw. The non-backdrivable nature of the motor drive system effectively decouples the motor from the applied force. Therefore, during joint loading, the motor sees the increasing torque required to turn the lead screw. The motor is capable of supplying the torque required to turn the lead screw during normal loading. However, thermal constraints limit the motor's endurance at high torque. To accommodate this constraint, the controller incorporates force feedback from the strain gauges installed on the lead screw shell. The controller utilizes the non-backdrivability of the motor drive system and properly turn's down motor output torque once a desired force is attained. During a grasp, a command to move in a direction that will increase the force beyond the 
desired level is ignored. If the force drops off or a command in a direction that will relieve the force is issued, the motor reverts to normal position control operation. This control strategy successfully lowers motor heating to acceptable levels and reduces power consumption

To perform joint control, the kinematics, which relates motor output to joint output, must be determined. As stated earlier, due to varying cable interactions a closed form kinematics algorithm is not tractable. Once the finger joint hall-effect based position sensors are calibrated using a resolver, a semi-autonomous kinematic calibration procedure for both forward and inverse kinematics is used to build look-up tables. Variations between kinematics and hall-effect sensor outputs during operation are seen in regions where the pre-loading springs are not effective. Designs using different spring strategies are under development to resolve this problem. To enhance positioning accuracy, a closed loop finger joint position controller employing hall-effect sensor position feedback is used as part of this kinematic calibration procedure.

An important safety feature of the hand, its ability to passively close in response to a contact on the back of the fingers, causes problems for closed loop joint control during normal operation. Further refinement of the kinematic calibration and the strain gauge force sensors is required to reliably determine when the fingers are being uncontrollably closed. Once this information, along with a better model for the drive train dynamics is available, the joint controller can be modified to distinguish the loaded from the normal operating mode.

Although considerable work still needs to be done, joint control satisfactory for teleoperated control of the hand has been attained. For initial tests, the hand was controlled in joint mode from inputs derived from the Cyberglove $(8)$ worn by the operator. The Cyberglove uses bend sensors, which are interpreted by the Cyberglove electronics to determine the position of 18 actions of the operator's hand. Some of these actions are absolute positions of finger joints while other are relative motions between joints. The challenge is developing a mapping between the 18 absolute and relative joint positions determined by the Cyberglove and the 12 joints of the Robonaut hand. This mapping must result in the Robonaut hand tracking the operator's hand as well as possible. While some joints are directly mapped, others required heuristic algorithms to fuse data from several glove sensors to produce a hand joint position command. In conjunction with an automated glove calibration program, a satisfactory mapping is experimentally obtainable. Using these custom mappings, operators are able to successfully manipulate many EVA tool. Several example tool manipulations using the Robonaut hand under teleoperated control are shown in figures 11 and 12 .
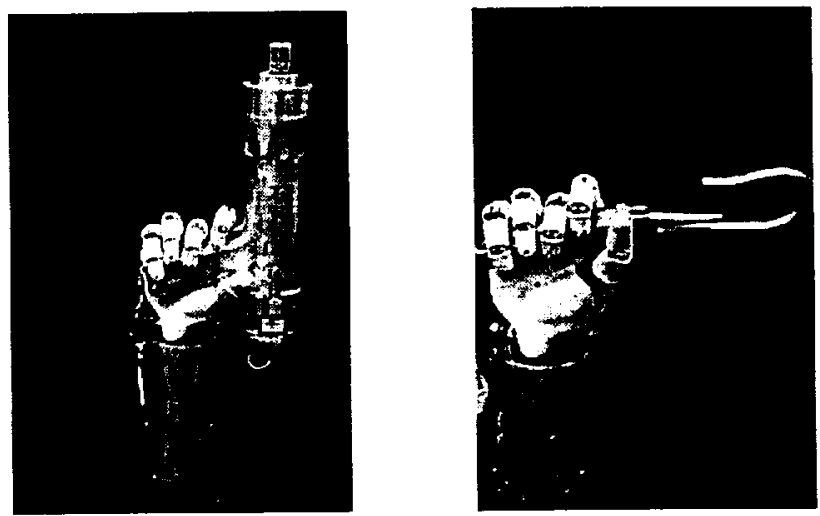

Figure 11: Examples of the Robonaut Hand using enveloping power grasps to hold tools
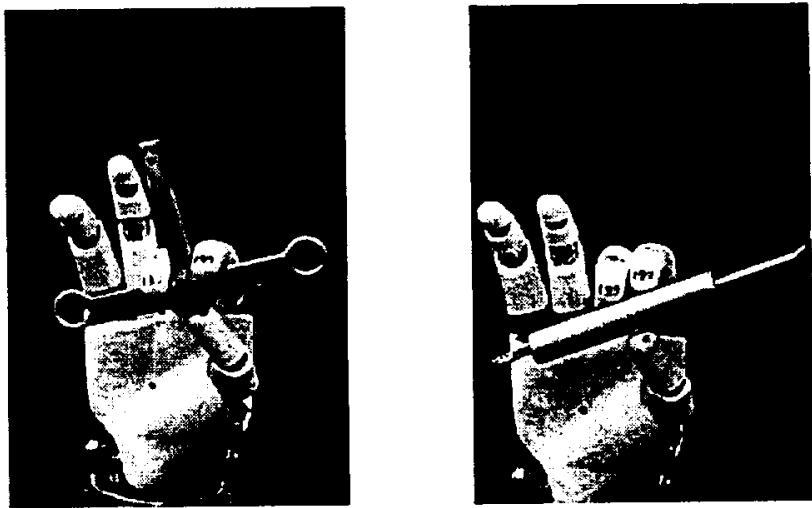

Figure 12: Examples of the Robonaut Hand using dexterous grasps for fine tool maipulation

To facilitate testing of the hand base level pads as shown in figures 11,12 were fabricated from Dow Cornings Silastic $\circledast$ E. The pads provide a nonslip compliant surface necessary for positively grasping an object. These pads will serve as the foundation for tactile sensors and be covered with a protective glove.

Future plans include the development of a grasp criteria measure for the stability of the hand grasp. These criteria will be used to assist the operator in determining if a grasp is acceptable. Since the baseline operation plan does not involve force feedback to the operator, visual feedback only may be insufficient to properly determine if a grasp is stable. Using some knowledge of the object which is being grasped in conjunction with the existing lead screw force sensors and a small set of additional tactile sensors installed on the fingers and palm, the control system will determine the acceptability of the grasp and indicate that measure to the operator. The operator can then decide how best to use this data in reconfiguring the grasp to a 
more stable configuration. This grasp criteria measure could evolve into an important part of an autonomous grasping system.

\section{Conclusions}

The Robonaut Hand is presented. This highly anthropomorphic human scale hand built at the NASA Johnson Space Center is designed to interface with EVA crew interfaces thereby increasing the number of robotically compatible operations available to the International Space Station. Several novel mechanisms are described that allow the Robonaut hand to achieve capabilities approaching that of an astronaut wearing a pressurized space suited glove. The initial joint based control strategy is discussed and example tool manipulations are illustrated.

\section{References}

1. Lovchik, C. S., Diftler, M. A., Compact Dexterous Robotic Hand. Patent Pending.

2. Salisbury, J. K., \& Mason, M. T., Robot Hands and the Mechanics of Manipulation. MIT Press, Cambridge, MA, 1985.

3. Jacobsen, S., et al., Design of the UtahM.I.T. Dextrous Hand. Proceedings of the IEEE International Conference on Robotics and Automation, San Francisco, CA, 1520-1532, 1986.

4. Bekey, G., Tomovic, R., Zeljkovic, I., Control Architecture for the Belgrade/USC Hand. Dexterous Robot Hands, 136-149, Springer-Verlag, New York, 1990.

5. Maeda, Y., Susumu, T., Fujikawa, A., Development of an Anthropomorphic Hand (Mark-1). Proceedings of the $20^{\text {th }}$ International Symposium on Industrial Robots, Tokyo, Japan, 53-544, 1989.

6. Ali, M., Puffer, R., Roman, H., Evaluation of a Multifingered Robot Hand for Nuclear Power Plant Operations and Maintenance Tasks. Proceedings of the $5^{\text {th }}$ World Conference on Robotics Research, Cambridge, MA, MS94-217, 1994.

7. Hartsfield, J., Smart Hands: Flesh is Inspiration for Next Generation of Mechanical Appendages. Space News Roundup, NASA Johnson Space Center, 27(35), page 3, Houston, TX, 1988.

8. Carter, E. Monford, G., Dexterous End Effector Flight Demonstration, Proceedings of the Seventh Annual Workshop on Space Operations Applications and Research, Houston, TX, 95-102, 1993.

9. Nagatomo, M. et al, On the Results of the MFD Flight Operations, Press Release, National Space Development Agency of Japan, August, 1997.
10. Stieber, M., Trudel, C., Hunter, D., Robotic systems for the International Space Station, Proceedings of the IEEE International Conference on Robotics and Automation, Albuquerque, New Mexico, 3068-3073, 1997.

11. Hirzinger, G., Brunner, B., Dietrich, J., Heindl, J., Sensor Based Space Robotics - ROTEX and its Telerobotic Features, IEEE Transactions on Robotics and Automation, 9(5), 649-663, 1993.

12. Akin, D., Cohen, R., Development of an Interchangeable End Effector Mechanism for the Ranger Telerobotic Vehicle., Proceedings of the $28^{\text {th }}$ Aerospace Mechanism Symposium, Cleveland $\mathrm{OH}$, $79-89,1994$

13. Jau, B., Dexterous Tele-manipulation with Four Fingered Hand System. Proceedings of the IEEE International Conference on Robotics and Automation, Nagoya, Japan, 338-343, 1995.

14. Butterfass, J., Hirzinger, G., Knoch, S. Liu, H., DLR's Multi-sensory Articulated Hand Part I: Hardand Software Architecture. Proceedings of the IEEE International Conference on Robotics and Automation, Leuven Belgium, 2081-2086, 1998.

15. Extravehicular Activity (EVA) Hardware Generic Design Requirements Document, JSC 26626, NASAJJohnson Space Center, Houston, Texas, July, 1994.

16. Shimoga, K.B., Robot Grasp Synthesis: A Survey, International Journal of Robotics Research, vol. 15, no. 3, pp. 230-266, 1996.

17. Mirza, K. and Orin, D., General Formulation for Force Distribution in Power Grasp, Proceedings of the IEEE International Conference on Robotics and Automation, p.880-887, 1994.

18. Li, L., Cox, B., Diftler, M., Shelton, S. , Rogers, B., Development of a Telepresence Controlled Ambidextrous Robot for Space Applications. Proceedings of the IEEE International Conference on Robotics and Automation, Minneapolis, MN, 58-63, 1996.

19. Li, L., Taylor, E., EWS Robonaut: Work in Progress, Proceedings of the International Symposium on Artificial Intelligence, Robotics and Automation in Space, Tokyo, Japan, T-8-4, 1997. 